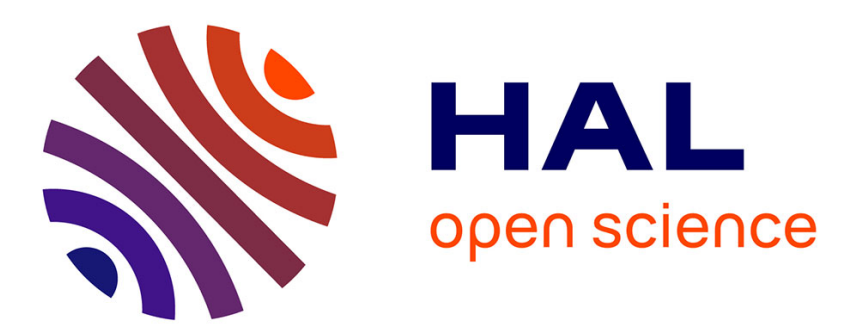

\title{
Exponential decay to equilibrium of nonlinear DDFV schemes for convection-diffusion equations
}

Claire Chainais-Hillairet, Stella Krell

\section{To cite this version:}

Claire Chainais-Hillairet, Stella Krell. Exponential decay to equilibrium of nonlinear DDFV schemes for convection-diffusion equations. FVCA 2020 - 9th Conference on Finite Volumes for Complex Applications, Jun 2020, Bergen, Norway. hal-02408212

\section{HAL Id: hal-02408212 \\ https://hal.science/hal-02408212}

Submitted on 12 Dec 2019

HAL is a multi-disciplinary open access archive for the deposit and dissemination of scientific research documents, whether they are published or not. The documents may come from teaching and research institutions in France or abroad, or from public or private research centers.
L'archive ouverte pluridisciplinaire HAL, est destinée au dépôt et à la diffusion de documents scientifiques de niveau recherche, publiés ou non, émanant des établissements d'enseignement et de recherche français ou étrangers, des laboratoires publics ou privés. 


\title{
Exponential decay to equilibrium of nonlinear DDFV schemes for convection-diffusion equations
}

\author{
Claire Chainais-Hillairet and Stella Krell
}

5 Abstract We introduce a nonlinear DDFV scheme for an anisotropic linear convectiondiffusion equation with mixed boundary conditions and we establish the exponential decay of the scheme towards its steady-state.

Key words: Discrete Duality Finite Volume scheme, discrete entropy/dissipation relation, long time behaviour.

10 MSC (2010): 65M08, 35B40.

\section{Motivation}

We are interested in the numerical discretization of linear anisotropic convectiondiffusion equations on almost general meshes. Let $\Omega$ be a polygonal connected open bounded subset of $\mathbb{R}^{2}$ and let $T>0$. The boundary $\Gamma=\partial \Omega$ is divided into two parts $\Gamma=\Gamma^{D} \cup \Gamma^{N}$ with $\mathrm{m}\left(\Gamma^{D}\right)>0$. The problem writes:

$$
\begin{aligned}
& \partial_{t} u+\operatorname{div} \mathbf{J}=0, \mathbf{J}=-\Lambda(\nabla u+u \nabla V) \text { in } \Omega \times(0, T), \\
& \mathbf{J} \cdot \mathbf{n}=0 \text { on } \Gamma^{N} \times(0, T), \text { and } u=u^{D} \text { on } \Gamma^{D} \times(0, T), \\
& u(\cdot, 0)=u_{0} \text { in } \Omega .
\end{aligned}
$$

We assume that the initial condition $u_{0}$ belong to $L^{\infty}(\Omega)$ and is positive, that the exterior potential $\mathrm{V}$ belongs to $C^{1}(\bar{\Omega}, \mathbb{R})$. The anisotropy tensor is supposed to be

Claire Chainais-Hillairet

Univ. Lille, CNRS,UMR 8524-Laboratoire Paul Painlevé. F-59000 Lille, France

e-mail: claire.chainais@univ-lille.fr

Stella Krell

Université Côte d'Azur, CNRS, Inria, LJAD, France

e-mail: stella.krell@univ-cotedazur.fr 
bounded, symmetric and uniformly elliptic : there exists $\lambda^{M} \geq \lambda_{m}>0$ such that

$$
\lambda_{m}|\mathbf{v}|^{2} \leq \Lambda(\mathbf{x}) \mathbf{v} \cdot \mathbf{v} \leq \lambda^{M}|\mathbf{v}|^{2} \quad \text { for all } \mathbf{v} \in \mathbb{R}^{2} \text { and almost all } \mathbf{x} \in \Omega .
$$

15 We finally assume that the boundary data $u^{D}$ corresponds to a thermal Gibbs equilibrium, which means the existence of $\rho>0$ such that $u^{D}=\rho e^{-V}$ on $\Gamma^{D}$. This implies that $u^{\infty}=\rho e^{-V}$ is a steady-state of (1). Moreover, the exponential decay of the solution $u$ to (1) towards $u^{\infty}$ is well-known, see for instance [2] (for even more general results) and the references therein.

When designing numerical schemes for (1), it is crucial to ensure that the scheme has a similar large time behavior than the continuous model. This property is ensured by classical TPFA schemes with linear B-fluxes on admissible meshes when $\Lambda=I$, as shown in [4]. Unfortunately, these schemes cannot be used in the anisotropic case and/or on general meshes. In [3], a nonlinear DDFV scheme has been introduced for (1) on general meshes in the case of Neumann boundary conditions. The convergence of the scheme has been proved and numerical experiments show the exponential decay of the scheme towards equilibrium. This last property has been recently established in [5]. The aim of this paper is to introduce the nonlinear DDFV scheme for (1) and to prove its exponential decay towards equilibrium. The main result is stated in Theorem 1.

\section{Presentation of the numerical scheme}

\subsection{Meshes and notations}

In order to define a DDFV scheme, we need to introduce three different meshes - the primal mesh, the dual mesh and the diamond mesh - and some associated notations, for more details and also illustrations see [1,3].

The primal mesh denoted $\overline{\mathfrak{M}}$ is composed of the interior primal mesh $\mathfrak{M}$ (a partition of $\Omega$ with polygonal control volumes) and the set $\partial \mathfrak{M}$ of boundary edges seen as degenerate control volumes. For all $K \in \overline{\mathfrak{M}}$, we define $x_{K}$ the center of $K$.

To any vertex $x_{K^{*}}$ of the primal mesh satisfying $x_{K^{*}} \in \Omega$, we associate a polygonal control volume $K^{*}$ defined by connecting all the centers of the primal cells sharing $x_{K^{*}}$ as vertex. The set of such control volumes is the interior dual mesh denoted $\mathfrak{M}^{*}$. To any vertex $x_{K^{*}} \in \partial \Omega$, we define a polygonal control volume $K^{*}$ by connecting the centers $x_{K}$ of the interior primal cells and the midpoints of the boundary edges sharing $x_{K^{*}}$ as vertex. The set of such control volumes is the boundary dual mesh, denoted $\partial \mathfrak{M}^{*}$. Finally, the dual mesh is $\mathfrak{M}^{*} \cup \partial \mathfrak{M}^{*}$, denoted by $\overline{\mathfrak{M}^{*}}$.

For all neighboring primal cells $K$ and $L$, we assume that $\partial K \cap \partial L$ is a segment, corresponding to an edge of the mesh $\mathfrak{M}$, denoted by $\sigma=K \mid L$. Let $\mathscr{E}$ be the set of such edges. We similarly define the set $\mathscr{E}^{*}$ of the edges of the dual mesh. For each couple $\left(\sigma, \sigma^{*}\right) \in \mathscr{E} \times \mathscr{E}^{*}$ such that $\sigma=K \mid L$ and $\sigma^{*}=K^{*} \mid L^{*}$, we define the quadrilateral diamond $\mathscr{D}_{\sigma, \sigma^{*}}$ whose diagonals are $\sigma$ and $\sigma^{*}$ (if $\sigma \subset \partial \Omega$, it degenerates 
into a triangle). The set of the diamonds defines the diamond mesh $\mathfrak{D}$, which is a partition of $\Omega$. Finally, the DDFV mesh is made of $\mathscr{T}=\left(\overline{\mathfrak{M}}, \overline{\mathfrak{M}^{*}}\right)$ and $\mathfrak{D}$.

For each primal cell $K \in \overline{\mathfrak{M}}$ and $K^{*} \in \overline{\mathfrak{M}^{*}}$, we define $\mathrm{m}_{K}$ the measure of $K, \mathrm{~m}_{K^{*}}$ the measure of $K^{*}$. For a diamond $\mathscr{D}=\mathscr{D}_{\sigma, \sigma^{*}}$, whose vertices are $\left(x_{K}, x_{K^{*}}, x_{L}, x_{L^{*}}\right)$, we define: $x_{\mathscr{D}}$ its center $\left(\left\{x_{\mathscr{D}}\right\}=\sigma \cap \sigma^{*}\right), \mathrm{m}_{\sigma}$ and $\mathrm{m}_{\sigma^{*}}$ the lengths of the edges, $\mathrm{m}_{\mathscr{D}}$ its measure, $d_{\mathscr{D}}$ its diameter, $\alpha_{\mathscr{D}}$ the angle between $\left(x_{K}, x_{L}\right)$ and $\left(x_{K^{*}}, x_{L^{*}}\right)$. We have $\mathrm{m}_{\mathscr{D}}=\frac{1}{2} \mathrm{~m}_{\sigma} \mathrm{m}_{\sigma^{*}} \sin \left(\alpha_{\mathscr{D}}\right)$. We will also use two direct basis $\left(\tau_{K^{*} L^{*}}, \mathbf{n}_{\sigma K}\right)$ and $\left(\mathbf{n}_{\sigma^{*} K^{*}}, \tau_{K, L}\right)$, where $\mathbf{n}_{\sigma K}$ is the unit normal to $\sigma$ outward $K, \mathbf{n}_{\sigma^{*} K^{*}}$ is the unit normal to $\sigma^{*}$ outward $K^{*}, \tau_{K^{*} L^{*}}$ is the unit tangent vector to $\sigma$, oriented from $K^{*}$ to $L^{*}, \tau_{K, L}$ is the unit tangent vector to $\sigma^{*}$, oriented from $K$ to $L$.

We define two local regularity factors $\theta_{\mathscr{D}}, \tilde{\theta}_{\mathscr{D}}$ of the diamond $\mathscr{D}$ by

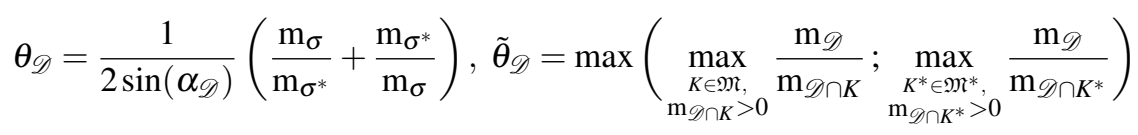

and we assume the following regularity of the mesh:

$$
\exists \Theta \geq 1 \text { such that } 1 \leq \theta_{\mathscr{D}}, \tilde{\theta}_{\mathscr{D}} \leq \Theta, \quad \forall \mathscr{D} \in \mathfrak{D} .
$$

Finally, we define the approximation $\Lambda^{\mathscr{D}}$ of the anisotropy tensor $\Lambda$ on each diamond $\mathscr{D} \in \mathfrak{D}$ as the mean value of $\Lambda$ over $\mathscr{D}$.

\subsection{Discrete unknowns and discrete operators}

We need several types of degrees of freedom to represent scalar and vector fields in the discrete setting. $\mathbb{R}^{\mathscr{T}}$ is the linear space of scalar fields constant on the primal and dual cells and $\left(\mathbb{R}^{2}\right)^{\mathfrak{D}}$ the linear space of vector fields constant on the diamonds:

$$
\begin{aligned}
u_{\mathscr{T}} \in \mathbb{R}^{\mathscr{T}} & \Longleftrightarrow u_{\mathscr{T}}=\left(\left(u_{K}\right)_{K \in \overline{\mathfrak{M}}},\left(u_{K^{*}}\right)_{K^{*} \in \overline{\mathfrak{M}^{*}}}\right) \\
\xi_{\mathfrak{D}} \in\left(\mathbb{R}^{2}\right)^{\mathfrak{D}} & \Longleftrightarrow \xi_{\mathfrak{D}}=\left(\xi_{\mathscr{D}}\right)_{\mathscr{D} \in \mathfrak{D}}
\end{aligned}
$$

We also define a positive semi-definite bilinear form on $\mathbb{R}^{\mathscr{T}}$ and a scalar product on $\left(\mathbb{R}^{2}\right)^{\mathfrak{D}}$ by

$$
\begin{aligned}
\llbracket v_{\mathscr{T}}, u_{\mathscr{T}} \rrbracket_{\mathscr{T}} & =\frac{1}{2}\left(\sum_{K \in \mathfrak{M}} \mathrm{m}_{K} u_{K} v_{K}+\sum_{K^{*} \in \overline{M^{*}}} \mathrm{~m}_{K^{*}} u_{K^{*}} v_{K^{*}}\right), \forall u_{\mathscr{T}}, v_{\mathscr{T}} \in \mathbb{R}^{\mathscr{T}}, \\
\left(\xi_{\mathfrak{D}}, \varphi_{\mathfrak{D}}\right)_{\Lambda, \mathfrak{D}} & =\sum_{\mathscr{D} \in \mathfrak{D}} \mathrm{m}_{\mathscr{D}} \xi_{\mathscr{D}} \cdot \Lambda^{\mathscr{D}} \varphi_{\mathscr{D}}, \quad \forall \xi_{\mathfrak{D}}, \varphi_{\mathfrak{D}} \in\left(\mathbb{R}^{2}\right)^{\mathfrak{D}} .
\end{aligned}
$$

The associated norms are respectively denoted by $\|\cdot\|_{2, \mathscr{T}}$ and $\|\cdot\|_{\Lambda, \mathfrak{D}}$.

The DDFV method is based on the definitions of a discrete gradient $\nabla^{\mathcal{D}}$, of a discrete divergence $\operatorname{div}^{\mathscr{T}}$ and a duality formula (see [1]). Here we do not recall the def- 
inition of the discrete operators as we will use a compact form of the scheme, as in [3]. For $u_{\mathscr{T}} \in \mathbb{R}^{\mathscr{T}}$, we just define $\delta^{\mathfrak{D}} u_{\mathscr{T}}=\left(\delta^{\mathscr{D}} u_{\mathscr{T}}\right)_{\mathscr{D} \in \mathfrak{D}}$ by $\delta^{\mathscr{D}} u_{\mathscr{T}}=\left(\begin{array}{c}u_{K}-u_{L} \\ u_{K^{*}}-u_{L^{*}}\end{array}\right)$ for all $\mathscr{D} \in \mathfrak{D}$. Then, the usual definition of the discrete gradient ensures that :

$$
\left(\nabla^{\mathfrak{D}} u_{\mathscr{T}}, \nabla^{\mathfrak{D}} v_{\mathscr{T}}\right)_{\Lambda, \mathfrak{D}}=\sum_{\mathscr{D} \in \mathfrak{D}} \delta^{\mathscr{D}} u_{\mathscr{T}} \cdot \mathbb{A}^{\mathscr{D}} \delta^{\mathscr{D}} v_{\mathscr{T}}, \quad \forall u_{\mathscr{T}}, v_{\mathscr{T}} \in \mathbb{R}^{\mathscr{T}},
$$

where

$$
\mathbb{A}^{\mathscr{D}}=\frac{1}{4 \mathrm{~m}_{\mathscr{D}}}\left(\begin{array}{cc}
\mathrm{m}_{\sigma}^{2} \mathbf{n}_{\sigma K} \cdot \Lambda^{\mathscr{D}} \mathbf{n}_{\sigma K} & \mathrm{~m}_{\sigma} \mathrm{m}_{\sigma^{*}} \mathbf{n}_{\sigma K} \cdot \Lambda^{\mathscr{D}} \mathbf{n}_{\sigma^{*} K^{*}} \\
\mathrm{~m}_{\sigma} \mathrm{m}_{\sigma^{*}} \mathbf{n}_{\sigma K} \cdot \Lambda^{\mathscr{D}} \mathbf{n}_{\sigma^{*} K^{*}} & \mathrm{~m}_{\sigma^{*}}^{2} \mathbf{n}_{\sigma^{*} K^{*}} \cdot \Lambda^{\mathscr{D}} \mathbf{n}_{\sigma^{*} K^{*}}
\end{array}\right) \quad \forall \mathscr{D} \in \mathfrak{D} .
$$

Finally, we introduce a reconstruction operator on diamonds $r^{\mathfrak{D}}$. It is a mapping from $\mathbb{R}^{\mathscr{T}}$ to $\mathbb{R}^{\mathfrak{D}}$ defined for all $u_{\mathscr{T}} \in \mathbb{R}^{\mathscr{T}}$ by $r^{\mathfrak{D}} u_{\mathscr{T}}=\left(r^{\mathscr{D}} u_{\mathscr{T}}\right)_{\mathscr{D} \in \mathfrak{D}}$, where for $\mathscr{D} \in$ $\mathfrak{D}$, whose vertices are $x_{K}, x_{L}, x_{K^{*}}, x_{L^{*}}, r^{\mathscr{D}} u_{\mathscr{T}}=\frac{1}{4}\left(u_{K}+u_{L}+u_{K^{*}}+u_{L^{*}}\right)$.

\subsection{The scheme}

A nonlinear DDFV scheme for the convection-diffusion equation with Neumann boundary conditions has already been introduced and analyzed in [3]. In this paper, we want to take into account Dirichlet boundary conditions on the part $\Gamma^{D}$ of the boundary. Let us introduce the set of Dirichlet boundary primal and dual cells :

$$
\partial \mathfrak{M}_{D}=\left\{K \in \partial \mathfrak{M}: K \subset \Gamma_{D}\right\}, \quad \partial \mathfrak{M}_{D}^{*}=\left\{K^{*} \in \partial \mathfrak{M}^{*}: x_{K^{*}} \in \bar{\Gamma}_{D}\right\},
$$

Then, for a given $v \in C\left(\Gamma^{D}\right)$, we define

$$
\mathrm{E}_{v}^{\Gamma_{D}}=\left\{u_{\mathscr{T}} \in \mathbb{R}^{\mathscr{T}} \text {, s. t. } \forall K \in \partial \mathfrak{M}_{D}, u_{K}=v\left(x_{K}\right) \text { and } \forall K^{*} \in \partial \mathfrak{M}_{D}^{*}, u_{K^{*}}=v\left(x_{K^{*}}\right)\right\} \text {. }
$$

Let $\Delta t$ be a time step. We first discretize the initial condition by taking the mean values of $u_{0}$ on the primal and dual cells and the exterior potential $V$ by taking its nodal values on the primal and dual cells. It defines $u_{\mathscr{T}}^{0}$ and $V_{\mathscr{T}}$. Then, for all $n \geq 0$, we look for $u_{\mathscr{T}}^{n+1} \in \mathrm{E}_{u^{D}}^{\Gamma_{D}}$ solution to:

$$
\begin{gathered}
\llbracket \frac{u_{\mathscr{T}}^{n+1}-u_{\mathscr{T}}^{n}}{\Delta t}, \psi_{\mathscr{T}} \rrbracket_{\mathscr{T}}+T_{\mathfrak{D}}\left(u_{\mathscr{T}}^{n+1} ; g_{\mathscr{T}}^{n+1}, \psi_{\mathscr{T}}\right)=0, \quad \forall \psi_{\mathscr{T}} \in \mathrm{E}_{0}^{I_{D}}, \\
T_{\mathfrak{D}}\left(u_{\mathscr{T}}^{n+1} ; g_{\mathscr{T}}^{n+1}, \psi_{\mathscr{T}}\right)=\sum_{\mathscr{D} \in \mathfrak{D}} r^{\mathscr{D}} u_{\mathscr{T}}^{n+1} \delta^{\mathscr{D}} g_{\mathscr{T}}^{n+1} \cdot \mathbb{A}^{\mathscr{D}} \delta^{\mathscr{D}} \psi_{\mathscr{T}}, \\
g_{\mathscr{T}}^{n+1}=\log \left(u_{\mathscr{T}}^{n+1}\right)+V_{\mathscr{T}} .
\end{gathered}
$$

The scheme is written here under a compact form. But it can also be expanded on primal and dual meshes after the introduction of conservative numerical fluxes. 


\section{Main results}

\section{Steady-state}

As the boundary conditions are at thermal equilibrium, $u^{D}=\rho e^{-V}$ on $\Gamma^{D}$ with $\rho>0$. Then, $u_{\mathscr{T}}^{\infty}=\rho e^{-V_{\mathscr{T}}}$ belongs to $\mathrm{E}_{u^{D}}^{\Gamma_{D}}$ and verifies $\delta^{\mathscr{D}}\left(\log u_{\mathscr{T}}^{\infty}+V_{\mathscr{T}}\right)=0$ for all $\mathscr{D} \in \mathfrak{D}$, so that it is a steady-state to the scheme (3).

Let us remark that, due to the definition of the steady-state, it is clearly bounded: there exists $m^{\infty}>0$ and $M^{\infty}>0$, such that $m^{\infty} \leq u_{\mathscr{T}}^{\infty} \leq M^{\infty}$.

\section{Entropy-dissipation estimate}

Let $\Phi_{1}: x \mapsto x \log x-x+1$ the Gibbs entropy. We define the discrete relative entropy $\left(\mathbb{E}_{1, \mathscr{T}}^{n}\right)_{n \geq 0}$ and its associated discrete dissipation $\left(\mathbb{I}_{1, \mathscr{T}}^{n+1}\right)_{n \geq 0}$ by:

$$
\begin{aligned}
& \mathbb{E}_{1, \mathscr{T}}^{n}=\llbracket u_{\mathscr{T}}^{\infty} \Phi_{1}\left(\frac{u_{\mathscr{T}}^{n}}{u_{\mathscr{T}}^{\infty}}\right), 1_{\mathscr{T}} \rrbracket, \quad \forall n \geq 0 \\
& \mathbb{I}_{1, \mathscr{T}}^{n+1}=T_{\mathfrak{D}}\left(u_{\mathscr{T}}^{n+1} ; g_{\mathscr{T}}^{n+1}, g_{\mathscr{T}}^{n+1}\right), \quad \forall n \geq 0
\end{aligned}
$$

The definition of the steady-state implies that $\delta^{\mathfrak{D}} g_{\mathscr{T}}^{n+1}=\delta^{\mathfrak{D}} \log \left(u_{\mathscr{T}}^{n+1} / u_{\mathscr{T}}^{\infty}\right)$, so that 80 $\mathbb{I}_{1, \mathscr{T}}^{n+1}$ rewrites

$$
\mathbb{I}_{1, \mathscr{T}}^{n+1}=\sum_{\mathscr{D} \in \mathfrak{D}} r^{\mathscr{D}}\left(u_{\mathscr{T}}^{n+1}\right) \delta^{\mathscr{D}} \log \left(\frac{u_{\mathscr{T}}^{n+1}}{u_{\mathscr{T}}^{\infty}}\right) \cdot \mathbb{A}^{\mathscr{D}} \delta^{\mathscr{D}} \log \left(\frac{u_{\mathscr{T}}^{n+1}}{u_{\mathscr{T}}^{\infty}}\right), \quad \forall n \geq 0
$$

Proposition 1. Let assume that the scheme (3) has a solution $u_{\mathscr{T}}^{n+1} \in \mathrm{E}_{u_{D}^{D}}^{\Gamma_{D}}$ for all $n \geq$ 0 , satisfying moreover $u_{\mathscr{T}}^{n+1}>0$. Then, the following entropy-dissipation estimate holds:

$$
\frac{\mathbb{E}_{1, \mathscr{T}}^{n+1}-\mathbb{E}_{1, \mathscr{T}}^{n}}{\Delta t}+\mathbb{I}_{1, \mathscr{T}}^{n+1} \leq 0, \text { for all } n \geq 0
$$

Proof. Due to the convexity of $\Phi_{1}$ and the fact that $\Phi_{1}^{\prime}(x)=\log x$, we have

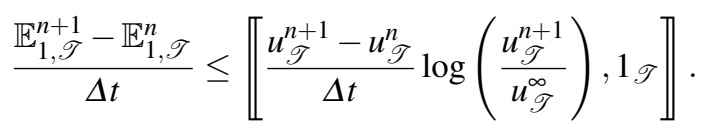

Then, we obtain (5) by taking $\psi_{\mathscr{T}}=\log \left(u_{\mathscr{T}}^{n+1} / u_{\mathscr{T}}^{\infty}\right) \in \mathrm{E}_{0}^{\Gamma_{D}}$ in the scheme (3).

As a consequence of the entropy-dissipation estimate, we may obtain the existence of a solution to the scheme (3). The result is a consequence of the control of the dissipation implied by (5) and of a topological degree argument. We refer to [3] for the idea of the proof. For the study of the exponential decay, we will further assume that the solution to the scheme satisfies uniform bounds. 


\section{Exponential decay}

Theorem 1. Assume that the solution to the scheme (3) is uniformly bounded:

$$
\exists m_{*} \in\left(0, m^{\infty}\right] \text { and } M^{*} \in\left[M^{\infty},+\infty\right) \text { such that } m_{*} \leq u_{\mathscr{T}}^{n} \leq M^{*} \quad \forall n \geq 0 .
$$

Then, there exists $v$ depending only $\Omega, \Theta, m_{*}, M^{*}$ and $\Lambda$, such that, for any $k>0$, if $\Delta t \leq k$,

$$
\mathbb{E}_{1, \mathscr{T}}^{n} \leq e^{-\tilde{v} t^{n}} \mathbb{E}_{1, \mathscr{T}}^{0}, \forall n \geq 0, \text { with } \tilde{v}=\frac{1}{k} \log (1+v k)
$$

Proof. Based on the entropy-dissipation estimate (5), the proof consists in establishing the existence of some $v>0$ such that

$$
\mathbb{I}_{1, \mathscr{T}}^{n+1} \geq v \mathbb{E}_{1, \mathscr{T}}^{n+1}, \quad \forall n \geq 0
$$

Using the definition of $\Phi_{1}, \mathbb{E}_{1, \mathscr{T}}^{n+1}$ rewrites: $\mathbb{E}_{1, \mathscr{T}}^{n+1}=\llbracket u_{\mathscr{T}}^{n+1} \log \left(u_{\mathscr{T}}^{n+1} / u_{\mathscr{T}}^{\infty}\right)-u_{\mathscr{T}}^{n+1}+$ $u_{\mathscr{T}}^{\infty}, 1 \mathscr{T} \rrbracket$. As $x \log (x / y)-x+y \leq(x-y)^{2} /(2 \min (x, y))$ for all $x, y>0$, we obtain:

$$
\mathbb{E}_{1, \mathscr{T}}^{n+1} \leq \frac{1}{2 m_{*}}\left\|u_{\mathscr{T}}^{n+1}-u_{\mathscr{T}}^{\infty}\right\|_{2, \mathscr{T}}^{2}, \quad \forall n \geq 0
$$

For all $\mathscr{D} \in \mathfrak{D}$, we introduce the diagonal matrix $\mathbb{B}^{\mathscr{D}}$, whose diagonal coefficients are $B_{\sigma, \sigma}^{\mathscr{D}}=\left|A_{\sigma, \sigma}^{\mathscr{D}}\right|+\left|A_{\sigma, \sigma^{*}}^{\mathscr{D}}\right|$ and $B_{\sigma^{*}, \sigma^{*}}^{\mathscr{D}}=\left|A_{\sigma^{*}, \sigma^{*}}^{\mathscr{D}}\right|+\left|A_{\sigma, \sigma^{*}}^{\mathscr{D}}\right|$. As shown in [3], there exists a constant $C(\Theta, \Lambda)$ depending only on $\Theta, \lambda_{m}$ and $\lambda^{M}$ such that

$$
\mathbf{w} \cdot \mathbb{A}^{D} \mathbf{w} \leq \mathbf{w} \cdot \mathbb{B}^{D} \mathbf{w} \leq C(\Theta, \Lambda) \mathbf{w} \cdot \mathbb{A}^{D} \mathbf{w}, \quad \forall \mathbf{w} \in \mathbb{R}^{2}, \forall \mathscr{D} \in \mathfrak{D}
$$

But, as $\mathbb{B}^{\mathscr{D}}$ is a diagonal matrix, for all $\mathscr{D} \in \mathfrak{D}$ we have:

$$
\begin{array}{r}
\delta^{\mathscr{D}} \log \frac{u_{\mathscr{T}}^{n+1}}{u_{\mathscr{T}}^{\infty}} \cdot \mathbb{B}^{\mathscr{D}} \delta^{\mathscr{D}} \log \frac{u_{\mathscr{T}}^{n+1}}{u_{\mathscr{T}}^{\infty}}=B_{\sigma, \sigma}^{\mathscr{D}}\left(\log \frac{u_{K}^{n+1}}{u_{K}^{\infty}}-\log \frac{u_{L}^{n+1}}{u_{L}^{\infty}}\right)^{2} \\
+B_{\sigma^{*}, \sigma^{*}}^{\mathscr{D}}\left(\log \frac{u_{K^{*}}^{n+1}}{u_{K^{*}}^{\infty}}-\log \frac{u_{L^{*}}^{n+1}}{u_{L^{*}}^{\infty}}\right)^{2} .
\end{array}
$$

As $(\log x-\log y)^{2} \geq(x-y)^{2} / \max ^{2}(x, y)$ for all $x, y>0$, we deduce from (6) that

$$
\delta^{\mathscr{D}} \log \frac{u_{\mathscr{T}}^{n+1}}{u_{\mathscr{T}}^{\infty}} \cdot \mathbb{B}^{\mathscr{D}} \delta^{\mathscr{D}} \log \frac{u_{\mathscr{T}}^{n+1}}{u_{\mathscr{T}}^{\infty}} \geq\left(\frac{m_{*}}{M^{*}}\right)^{2} \delta^{\mathscr{D}} \frac{u_{\mathscr{T}}^{n+1}}{u_{\mathscr{T}}^{\infty}} \cdot \mathbb{B}^{\mathscr{D}} \delta^{\mathscr{D}} \frac{u_{\mathscr{T}}^{n+1}}{u_{\mathscr{T}}^{\infty}}
$$

From (4), (6), (10) (applied twice) and (2), we deduce that

$$
\mathbb{I}_{1, \mathscr{T}}^{n+1} \geq C(\Theta, \Lambda) m_{*}\left(\frac{m_{*}}{M^{*}}\right)^{2} \lambda_{m}\left\|\nabla^{\mathfrak{D}} \frac{u_{\mathscr{T}}^{n+1}}{u_{\mathscr{T}}^{\infty}}\right\|_{I, \mathfrak{D}}^{2}
$$


Let us now apply the discrete Poincaré inequality to $u_{\mathscr{T}}^{n+1} / u_{\mathscr{T}}^{\infty}-1 \in \mathrm{E}_{0}^{\Gamma_{D}}$ (see [1]). Combined with (6), this yields

$$
\left\|u_{\mathscr{T}}^{n+1}-u_{\mathscr{T}}^{\infty}\right\|_{2, \mathscr{T}}^{2} \leq C_{P}(\Omega) M^{* 2}\left\|\nabla^{\mathfrak{D}} \frac{u_{\mathscr{T}}^{n+1}}{u_{\mathscr{T}}^{\infty}}\right\|_{I, \mathfrak{D}}^{2} .
$$

From (9), (11), (12), we finally deduce (8), with

$$
v=C(\Theta, \Lambda, \Omega)\left(\frac{m_{*}}{M^{*}}\right)^{4} .
$$

This concludes the proof of Theorem 1.

\section{Numerical experiments}

We consider a test case where $\Omega=(0,1)^{2}, V\left(x_{1}, x_{2}\right)=-x_{1}, \Gamma_{D}=\left\{x_{1}=0\right\} \cup\left\{x_{1}=\right.$ $1\}$ and the exact solution $u_{\text {ex }}$ is defined by

$$
u_{\mathrm{ex}}\left(\left(x_{1}, x_{2}\right), t\right)=e^{-\alpha t+\frac{x_{1}}{2}} \sin \left(\pi x_{1}\right)+e^{x_{1}}
$$

with $\alpha=\pi^{2}+\frac{1}{4}$. We choose $u_{0}=u_{\mathrm{ex}}(\cdot, 0)$.

In order to illustrate the convergence and the robustness of our method, we test its convergence on two sequences of meshes. The first sequence of primal meshes is made of successively refined Kershaw meshes. The second sequence of primal meshes is the so-called quadrangle meshes mesh_quad_i of the FVCA8 benchmark on incompressible flows. In the refinement procedure, the time step is divided by 4 when the mesh size is divided by 2 .

The nonlinear system (3) is solved thanks to Newton's method. In order to avoid the singularity of the $\log$ near 0 , the sequence $\left(u_{\mathscr{T}}^{n+1, i}\right)_{i>0}$ to compute $u_{\mathscr{T}}^{n+1}$ from the previous state $\left(u_{\mathscr{T}}^{n}\right)_{i>0}$ is initialized by $u_{\mathscr{T}}^{n+1,0}=\max \left(u_{\mathscr{T}}^{n}, 10^{-12}\right)$. As a stopping criterion, we require the $\ell^{1}$-norm of the residual to be smaller than $10^{-10}$. In Table 1 , the quantities erru and errgu respectively denote the $L^{\infty}\left((0, T) ; L^{2}(\Omega)\right)$ error on the solution and the $L^{2}(\Omega \times(0, T))^{2}$ error on the gradient, whereas ordu and ordgu are the corresponding convergence orders. It appears that the method is second order accurate w.r.t. space.

The maximal (resp. mean) number of Newton iterations by time step is denoted by $N_{\max }$ (resp. $N_{\text {mean }}$ ). We observe that the needed number of Newton iterations starts from a reasonably small value and falls down to 1 after a small number of time steps. Therefore, our method does not imply an important extra computational cost when compared to linear methods. Eventually, we observe numerically that the numerical solution remains bounded in time along the simulation (the bounds are reached at the initial time), which validates the hypothesis (6) of Theorem 1. 


\begin{tabular}{|c|c||c|c|c|c|c|c|c|c|}
\hline $\mathrm{M}$ & $\mathrm{dt}$ & errgu & ordgu & erru & ordu & $N_{\max }$ & $N_{\text {mean }}$ & $\operatorname{Min} u^{n}$ & $\operatorname{Max} u^{n}$ \\
\hline 1 & $1.613 \mathrm{E}-03$ & $3.447 \mathrm{E}-02$ & - & $5.208 \mathrm{E}-03$ & - & 2 & 2 & 1.0 & 3.148 \\
\hline 2 & $4.032 \mathrm{E}-04$ & $1.578 \mathrm{E}-02$ & 1.12 & $1.389 \mathrm{E}-03$ & 1.90 & 2 & 2 & 1.0 & 3.161 \\
\hline 3 & $1.008 \mathrm{E}-04$ & $8.629 \mathrm{E}-03$ & 0.92 & $4.467 \mathrm{E}-04$ & 1.72 & 2 & 2 & 1.0 & 3.161 \\
\hline 4 & $2.520 \mathrm{E}-05$ & $3.934 \mathrm{E}-03$ & 1.19 & $1.157 \mathrm{E}-04$ & 2.04 & 2 & 1 & 1.0 & 3.162 \\
\hline 5 & $6.300 \mathrm{E}-06$ & $9.668 \mathrm{E}-04$ & 1.66 & $2.402 \mathrm{E}-05$ & 1.86 & 1 & 1 & 1.0 & 3.162 \\
\hline
\end{tabular}

Table 1 Numerical results on the Quadrangle mesh family, final time $\mathrm{T}=0.1$.

In order to give an evidence of the good large-time behavior of our scheme, we plot in Figure 1 the evolution of the discrete relative entropy $\mathbb{E}_{1, \mathscr{T}}^{n}$ computed on the Kershaw and Quadrangle meshes. We observe the exponential decay of the relative energy.
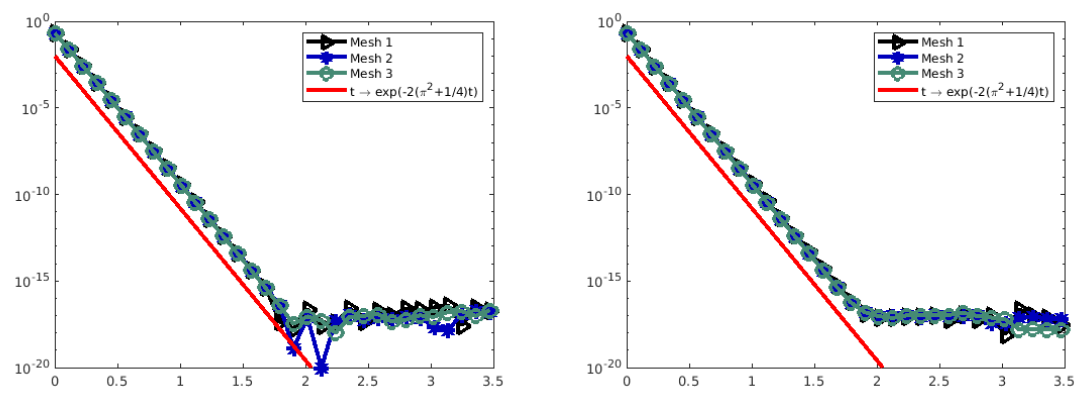

Fig. 1 Discrete relative energy $\mathbb{E}_{\mathscr{T}}^{n}-\mathbb{E}_{\mathscr{T}}^{\infty}$ as a function of $n \Delta t$. Left : computed on the first three Quadrangle meshes. Right: computed on the first three Kershaw meshes.

\section{References}

1. Andreianov, B., Boyer, F., Hubert, F.: Discrete duality finite volume schemes for Leray-Lionstype elliptic problems on general 2D meshes. Numer. Methods Partial Differential Equations 23(1), 145-195 (2007)

2. Bodineau, T., Lebowitz, J., Mouhot, C., Villani, C.: Lyapunov functionals for boundary-driven nonlinear drift-diffusion equations, Nonlinearity 27, 2111-2132 (2014)

3. Cancès, C., Chainais-Hillairet, C., Krell, S.: Numerical analysis of a nonlinear free-energy diminishing discrete duality finite volume scheme for convection diffusion equations. Comput. Methods Appl. Math., 18, 407-432 (2018)

4. Chainais-Hillairet, C., Herda, M.: Large-time behavior of a family of finite volume schemes for boundary-driven convection-diffusion equations. to appear in IMAJNA (2019)

5. Cancès, C., Chainais-Hillairet, C., Herda, M., Krell, S.: Large time behavior of nonlinear finite volume schemes for convection-diffusion equations. https://hal.archives-ouvertes.fr/hal02360155 (2019) 